\title{
Front Matter: Volume 6822
}

, "Front Matter: Volume 6822," Proc. SPIE 6822, Visual Communications and Image Processing 2008, 682201 (28 January 2008); doi: 10.1117/12.786987

SPIE. Event: Electronic Imaging, 2008, San Jose, California, United States 


\title{
Visual Communications and Image Processing 2008
}

\author{
William A. Pearlman \\ John W. Woods \\ Ligang Lu \\ Editors \\ 29-31 January 2008 \\ San Jose, California, USA \\ Sponsored and Published by \\ IS\&T-The Society for Imaging Science and Technology \\ SPIE \\ Cosponsored by \\ IBM Corporation (USA)
}


The papers included in this volume were part of the technical conference cited on the cover and title page. Papers were selected and subject to review by the editors and conference program committee. Some conference presentations may not be available for publication. The papers published in these proceedings reflect the work and thoughts of the authors and are published herein as submitted. The publishers are not responsible for the validity of the information or for any outcomes resulting from reliance thereon.

Please use the following format to cite material from this book:

Author(s), "Title of Paper," in Visual Communications and Image Processing 2008, edited by William A. Pearlman, John W. Woods, Ligang Lu, Proceedings of SPIE-IS\&T Electronic Imaging, SPIE Vol. 6822, Article CID Number (2008).

ISSN 0277-786X

ISBN 9780819469946

Copublished by

SPIE

P.O. Box 10, Bellingham, Washington $98227-0010$ USA

Telephone +1 3606763290 (Pacific Time) · Fax +1 3606471445

SPIE.org

and

IS\&T-The Society for Imaging Science and Technology

7003 Kilworth Lane, Springfield, Virginia, 22151 USA

Telephone +1 7036429090 (Eastern Time) · Fax +1 7036429094

imaging.org

Copyright @ 2008, Society of Photo-Optical Instrumentation Engineers and The Society for Imaging Science and Technology.

Copying of material in this book for internal or personal use, or for the internal or personal use of specific clients, beyond the fair use provisions granted by the U.S. Copyright Law is authorized by the publishers subject to payment of copying fees. The Transactional Reporting Service base fee for this volume is $\$ 18.00$ per article (or portion thereof), which should be paid directly to the Copyright Clearance Center (CCC), 222 Rosewood Drive, Danvers, MA 01923. Payment may also be made electronically through CCC Online at copyright.com. Other copying for republication, resale, advertising or promotion, or any form of systematic or multiple reproduction of any material in this book is prohibited except with permission in writing from the publisher. The CCC fee code is 0277-786X/08/ $\$ 18.00$.

Printed in the United States of America.

Paper Numbering: Proceedings of SPIE follow an e-First publication model, with papers published first online and then in print and on CD-ROM. Papers are published as they are submitted and meet publication criteria. A unique, consistent, permanent citation identifier (CID) number is assigned to each article at the time of the first publication. Utilization of CIDs allows articles to be fully citable as soon they are published online, and connects the same identifier to all online, print, and electronic versions of the publication. SPIE uses a six-digit CID article numbering system in which:

- The first four digits correspond to the SPIE volume number.

- The last two digits indicate publication order within the volume using a Base 36 numbering system employing both numerals and letters. These two-number sets start with 00, 01, 02, 03, 04, 05, 06, 07, 08 , $09,0 A, O B \ldots 0 Z$, followed by 10-1Z, 20-2Z, etc.

The CID number appears on each page of the manuscript. The complete citation is used on the first page, and an abbreviated version on subsequent pages. Numbers in the index correspond to the last two digits of the six-digit CID number. 


\section{Contents}

\section{Part One}

xi Conference Committee

\section{SESSION 1 MEDIA OVER NETWORKS}

682202 Measuring the Hurst parameter of compressed video sequences [6822-01]

M. E. Nilsson, S. Appleby, I. B. Crabtree, British Telecommunications plc (United Kingdom)

682203 Coalition-based multimedia peer matching strategies for P2P networks [6822-02]

H. Park, M. van der Schaar, Univ. of California, Los Angeles (USA)

682204 Risk-aware scheduling for multi-user video streaming over wireless multi-hop networks [6822-03]

H.-P. Shiang, M. van der Schaar, Univ. of California, Los Angeles (USA)

682205 Video multicast over wireless mesh networks with scalable video coding (SVC) [6822-04] X. Zhu, Stanford Univ. (USA); T. Schierl, T. Wiegand, Fraunhofer HHI - Heinrich Hertz Institut (Germany); B. Girod, Stanford Univ. (USA)

682206 Rate-distortion optimized multimedia communication in networks (Invited Paper) [6822-05] N. Sarshar, Univ. of Regina (Canada); X. Wu, McMaster Univ. (Canada)

682207 Distributed fine grain adaptive-FEC scheme for scalable video streaming [6822-06]

Y. Shan, J. W. Woods, S. Kalyanaraman, Rensselaer Polytechnic Institute (USA)

\section{SESSION 2 VIDEO PROCESSING}

682208 Motion-compensated noise estimation for effective video processing [6822-07]

B. C. Song, N. H. Kim, Samsung Electronics Co., Ltd. (South Korea)

682209 Motion tracking with non-stationary camera based on area and level set weighted average of centroid shifting vectors [6822-08]

S.-H. Lee, M. G. Kang, Yonsei Univ. (South Korea)

6822 0A A novel approach to skip mode decision for H.264 [6822-09]

D. Wu, K. P. Lim, W. Yao, T. K. Chiew, J. Y. Tham, Institute for Infocomm Research

(Singapore)

6822 OB Behavior subtraction [6822-10]

P.-M. Jodoin, Univ. de Sherbrooke (Canada); V. Saligrama, J. Konrad, Boston Univ. (USA)

6822 0C Distributed pose estimation from multiple views [6822-11]

C. Chen, D. Schonfeld, Univ. of Illinois, Chicago (USA); M. Mohamed, Motorola Labs. (USA) 
6822 OD Focused video estimation from defocused video sequences [6822-12]

J. Yang, D. Schonfeld, Univ. of Illinois, Chicago (USA); M. Mohamed, Motorola Labs. (USA)

6822 OE Resource management in particle filtering for multiple object tracking [6822-13]

P. Pan, D. Schonfeld, Univ. of Illinois, Chicago (USA)

\section{SESSION $3 \quad$ INDEXING AND RETRIEVAL}

6822 OF Exploring inter-frame correlation analysis and wavelet-domain modeling for real-time caption detection in streaming video [6822-15]

J. Li, Institute of Computing Technology (China) and Graduate Univ. of Chinese Academy of Sciences (China); Y. Tian, Peking Univ. (China); W. Gao, Institute of Computing Technology (China) and Peking Univ. (China)

$68220 \mathrm{G}$ Exploring the relationships of regions for visual content understanding [6822-16] T. Liu, Institute of Computing Technology (China) and Graduate Univ. of the Chinese Academy of Sciences (China); W. Wang, Graduate Univ. of Chinese Academy of Sciences (China); Y. Tian, T. Huang, Peking Univ. (China)

$6822 \mathrm{OH} \quad$ Efficient multi-ranking based on view selection for content based image retrieval [6822-17] F. Wang, Q. Dai, G. Er, Tsinghua Univ. (China)

$6822 \mathrm{Ol} \quad$ View-based 3D object retrieval and recognition using tangent subspace analysis [6822-18] F. Wang, F. Li, Q. Dai, G. Er, Tsinghua Univ. (China)

\section{SESSION 4 SCALABLE VIDEO CODING}

6822 0J Performance vs. complexity in scalable video coding for embedded surveillance applications [6822-20]

M. J. H. Loomans, VDG Security B.V. (Netherlands) and Eindhoven Univ. of Technology (Netherlands); C. J. Koeleman, VDG Security B.V. (Netherlands); P. H. N. de With, Eindhoven Univ. of Technology (Netherlands) and LogicaCMG (Netherlands)

6822 OK Error reduction in inter-layer motion prediction using FGS refined motion [6822-21] H. Yoo, D. S. Lee, S. H. Jin, T. M. Bae, Y. M. Ro, Information and Communications Univ. (South Korea)

$6822 \mathrm{OL} \quad$ An efficient block mode decision for temporal scalability in scalable video coding [6822-22]

B. Lee, M. Kim, Information and Communications Univ. (South Korea); S. Hahm, C. Park, K. Park, Korea Broadcasting System (South Korea)

$68220 \mathrm{M}$ Fast rate allocation based on distortion estimation modeling in scalable video coding [6822-23]

C. Gu, D. Zhao, Harbin Institute of Technology (China); X. Ji, Institute of Computing Technology (China)

6822 ON Smooth extraction of SVC fine-granular SNR scalable videos with a virtual-GOP-based rate distortion modeling [6822-24]

J. Sun, W. Gao, Peking Univ. (China); D. Zhao, Harbin Institute of Technology (China) 
682200 Bit-depth scalable coding for high dynamic range video [6822-25]

S. Liu, Mitsubishi Electric Research Labs. (USA); W.-S. Kim, Univ. of Southern California (USA);

A. Vetro, Mitsubishi Electric Research Labs. (USA)

6822 OP A new subband/wavelet framework for AVC/H.264 intraframe coding and performance comparison with Motion-JPEG2000 [6822-26]

S.-T. Hsiang, Motorola Labs. (USA)

SESSION 5

IMAGE/VIDEO TRANSMISSION

$6822 \mathrm{OQ}$ Optimal joint power-rate adaptation for error resilient video coding [6822-27]

Y. Lin, Norwegian Univ. of Science and Technology (Norway); E. Gürses, Univ. of Waterloo (Canada); A. N. Kim, A. Perkis, Norwegian Univ. of Science and Technology (Norway)

6822 OR Mobile video communications using a Wyner-Ziv transcoder [6822-28]

E. Peixoto, R. L. de Queiroz, Univ. de Brasilia (Brazil); D. Mukherjee, Hewlett-Packard Labs. (USA)

6822 OS Macroblock selection algorithms for error resilient H.264 video wireless transmission using redundant slices [6822-29]

P. Ferré, D. Agrafiotis, D. Bull, Univ. of Bristol (United Kingdom)

6822 OT Feedback-aided error resilience technique based on Wyner-Ziv coding [6822-30]

L. Liang, Purdue Univ. (USA); P. Salama, Indiana Univ.-Purdue Univ. Indianapolis (USA);

E. J. Delp, Purdue Univ. (USA)

6822 OU Real-time joint source-channel coding of multiple correlated substream progressive sources for multiple-antenna Rayleigh channels [6822-31]

M. Farshchian, Telephonics (USA); W. A. Pearlman, Rensselaer Polytechnic Institute (USA)

6822 OV Combating error bursts for enhanced video transmission using cross-packet FEC and description interleaving [6822-32]

M. Tesanovic, D. R. Bull, A. Doufexi, Univ. of Bristol (United Kingdom)

6822 OW Server-driven progressive image transmission of JPEG 2000 [6822-33]

D. Schwenke, A. Vetro, Mitsubishi Electric Research Labs. (USA); T. Hata, Mitsubishi Electric Corp. (Japan)

\section{SESSION 6 VIDEO CODING}

6822 OX Stochastic texture synthesis for video compression [6822-34]

S. de Waele, F. Zuo, Philips Research Europe (Netherlands)

6822 OY Decision trees for denoising in H.264/AVC video sequences [6822-35]

G. Huchet, J.-Y. Chouinard, Laval Univ. (Canada); D. Wang, A. Vincent, Communications Research Ctr. Canada (Canada)

$6822 \mathrm{OZ}$ Spatio-temporal fuzzy filtering for coding artifacts reduction [6822-36]

D. T. Võ, Univ. of California at San Diego (USA); S. Yea, A. Vetro, Mitsubishi Electric Research Labs. (USA) 
682210 Modeling quantization matrices for perceptual image/video encoding [6822-37]

H. Zhang, Cisco Systems, Inc. (USA); G. Cote, Mobilygen Corp. (USA)

682211 Determining optimal configuration of video encoding parameters using numerical search algorithms [6822-38]

H. Zhang, Cisco Systems, Inc. (USA); G. Cote, Mobilygen Corp. (USA)

682212 Video compression with tunable complexity via flexible syntax design [6822-39]

Y. Liu, D. Mukherjee, Hewlett-Packard Labs. (USA)

682213 Complexity constrained rate-distortion optimization of sign language video using an objective intelligibility metric [6822-40]

F. M. Ciaramello, S. S. Hemami, Cornell Univ. (USA)

682214 Focus mismatches in multiview systems and efficient adaptive reference filtering for multiview video coding [6822-41]

P. Lai, Univ. of Southern California (USA) and Thomson Corporate Research (USA);

A. Ortega, Univ. of Southern California (USA); P. Pandit, P. Yin, C. Gomila, Thomson

Corporate Research (USA)

682215 Fast H.264 mode selection using depth information for distributed game viewing [6822-42]

G. Cheung, Hewlett-Packard Labs. Japan (Japan); A. Ortega, Univ. of Southern California (USA); T. Sakamoto, Hewlett-Packard Labs. Japan (Japan)

\section{SESSION 7 SPECIAL SESSION: VLSI ARCHITECTURES}

682216 Optical, analog, and digital domain architectural considerations for visual communications (Invited Paper) [6822-43]

W. A. Metz, Texas Instruments Inc. (USA)

682217 Fast on-chip mean filter requiring only integer operations (Invited Paper) [6822-45] B. B. Bhattacharya, Indian Statistical Institute (India); A. Biswas, P. Bhowmick, Bengal Engineering and Science Univ. (India); T. Acharya, Avisere Inc. (USA)

682218 Implication of variable code block size in JPEG 2000 and its VLSI implementation (Invited Paper) [6822-46]

P.-S. Tsai, Univ. of Texas - Pan American (USA); T. Acharya, Avisere Inc. (USA) and Arizona State Univ. (USA)

682219 Parallel processing of multi-dimensional data with causal neighborhood dependencies [6822-47]

D. S. Turaga, K. Ratakonda, IBM T.J. Watson Research Ctr. (USA)

$68221 \mathrm{~A}$ Image processing assisted voltage overscaling for energy efficient IC realization of motion estimation (Invited Paper) [6822-48]

R. Dani, T. Zhang, J. W. Woods, Rensselaer Polytechnic Institute (USA) 


\section{Part Two}

\section{SESSION $8 \quad$ IMAGE PROCESSING}

6822 1B A new image denoising framework based on bilateral filter [6822-49]

M. Zhang, B. K. Gunturk, Louisiana State Univ. (USA)

6822 1C Multiband locally adaptive contrast enhancement algorithm with built-in noise and artifact suppression mechanisms [6822-50]

S. D. Cvetkovic, Bosch Security Systems (Netherlands) and Univ. of Technology

Eindhoven/LogicaCMG (Netherlands); J. Schirris, Bosch Security Systems (Netherlands);

P. H. N. de With, Univ. of Technology Eindhoven/LogicaCMG (Netherlands)

6822 1D An adaptive M-estimation framework for robust image super resolution without regularization [6822-51]

N. A. El-Yamany, P. E. Papamichalis, Southern Methodist Univ. (USA)

$68221 \mathrm{E} \quad$ Attraction-repulsion expectation maximization algorithm for image processing and sensor field networks [6822-52]

H. Hong, D. Schonfeld, Univ. of Illinois at Chicago (USA)

6822 IF Image segmentation and classification based on a 2D distributed hidden Markov model [6822-53]

X. Ma, D. Schonfeld, A. Khokhar, Univ. of Illinois at Chicago (USA)

$68221 \mathrm{H} \quad$ Line segment based image registration [6822-55]

Y. Li, R. L. Stevenson, J. Gai, Univ. of Notre Dame (USA)

\section{SESSION 9 SPECIAL SESSION: COLOR DEMOSAICKING I}

$68221 \mathrm{~J} \quad$ Image demosaicing: a systematic survey (Invited Paper) [6822-57]

X. Li, West Virginia Univ. (USA); B. Gunturk, Louisiana State Univ. (USA); L. Zhang, The Hong Kong Polytechnic Univ. (Hong Kong China)

6822 1K Denoising and interpolation of noisy Bayer data with adaptive cross-color filters (Invited Paper) [6822-58]

D. Paliy, A. Foi, Tampere Univ. of Technology (Finland); R. Bilcu, Nokia Research Ctr.

(Finland); V. Katkovnik, Tampere Univ. of Technology (Finland)

$68221 \mathrm{~L} \quad$ A regularization approach to demosaicking (Invited Paper) [6822-59]

D. Menon, G. Calvagno, Univ. of Padova (Italy)

$68221 \mathrm{M}$ Frequency selection demosaicking: a review and a look ahead (Invited Paper) [6822-60]

D. Alleysson, Lab. de Psychologie et NeuroCognition, CNRS (France); B. Chaix de

Lavarène, Grenoble Image Parole Signal Automatique, CNRS (France) 
$68221 \mathrm{~N}$ Improved color demosaicking in weak spectral correlation (Invited Paper) [6822-61] F. Zhang, Shanghai Jiaotong Univ. (China); X. Wu, McMaster Univ. (Canada); X. Yang, W. Zhang, Shanghai Jiaotong Univ. (China)

682210 Improved demosaicking in the frequency domain by restoration filtering of the LCC bands (Invited Paper) [6822-62]

M. Beermann, E. Dubois, Univ. of Ottawa (Canada)

$68221 \mathrm{P}$ Second-generation color filter array and demosaicking designs (Invited Paper) [6822-63] K. Hirakawa, P. J. Wolfe, Harvard Univ. (USA)

\section{SESSION 11 SPECIAL SESSION: DISTRIBUTED SOURCE CODING I}

$68221 Q \quad$ Balanced distributed coding of omnidirectional images (Invited Paper) [6822-64]

V. Thirumalai, I. Tosic, P. Frossard, Ecole Polytechnique Fédérale de Lausanne (Switzerland)

6822 IR A rate-efficient approach for establishing visual correspondences via distributed source coding (Invited Paper) [6822-65]

C. Yeo, P. Ahammad, K. Ramchandran, Univ. of California, Berkeley (USA)

6822 is Compression algorithms for flexible video decoding (Invited Paper) [6822-66]

N.-M. Cheung, A. Ortega, Univ. of Southern California (USA)

6822 IT Rate control algorithm for pixel-domain Wyner-Ziv video coding (Invited Paper) [6822-69] A. Roca, Univ. Politécnica de València (Spain); M. Morbée, Ghent Univ. (Belgium);

J. Prades-Nebot, Univ. Politécnica de València (Spain); E. J. Delp, Purdue Univ. (USA)

$68221 \mathrm{U}$ Wyner-Ziv video compression using rateless LDPC codes (Invited Paper) [6822-70]

D. He, A. Jagmohan, L. LU, V. Sheinin, IBM T.J. Watson Research Ctr. (USA)

\section{SESSION 12 SPECIAL SESSION: DISTRIBUTED SOURCE CODING II}

6822 IV Wyner-Ziv coding of 3D dynamic meshes (Invited Paper) [6822-67]

C. Chen, Q. Wang, Q. Dai, Tsinghua Univ. (China); Z. Xiong, Texas A\&M Univ. (USA); X. Liu, Tsinghua Univ. (China)

6822 IW Wyner-Ziv video coding with multi-resolution motion refinement: theoretical analysis and practical significance (Invited Paper) [6822-87]

W. Liu, L. Dong, W. Zeng, Univ. of Missouri, Columbia (USA)

$68221 \mathrm{X}$ Scalable Wyner-Ziv video coding with adaptive bit-plane representation (Invited Paper) [6822-88]

M. Guo, Harbin Institute of Technology (China); Y. Lu, F. WU, S. Li, Microsoft Research Asia

(China); W. Gao, Harbin Institute of Technology (China) 
$68221 Y \quad$ Region-based fusion strategy for side information generation in DMVC (Invited Paper) [6822-68]

Y. Li, Graduate Univ., Chinese Academy of Sciences (China); X. Ji, Institute of Computing Technology (China); D. Zhao, Harbin Institute of Technology (China); W. Gao, Peking Univ. (China)

$68221 \mathrm{Z}$ On the importance of source classification in Wyner-Ziv video coding (Invited Paper) [6822-71]

X. Li, West Virginia Univ. (USA)

\section{INTERACTIVE PAPER SESSION: IMAGE/VIDEO PROCESSING}

682220 Horizon detection based on sky-color and edge features [6822-72]

B. Zafarifar, NXP Research (Netherlands); H. Weda, Philips Research (Netherlands);

P. H. N. de With, Eindhoven Univ. of Technology (Netherlands)

682221 Efficient free viewpoint image acquisition from multiple differently focused images [6822-73]

X. Ou, Tokyo Univ. of Science (Japan) and National Institute of Informatics (Japan); T. Hamamoto, Tokyo Univ. of Science (Japan); A. Kubota, Tokyo Institute of Technology (Japan); K. Kodama, National Institute of Informatics (Japan)

682222 Multiframe image and video super-resolution algorithm with inaccurate motion registration errors rejection [6822-74]

O. A. Omer, T. Tanaka, Tokyo Univ. of Agriculture and Technology (Japan)

682223 Color transfer based on wavelet transform [6822-56]

K. Li, Q. Dai, W. Xu, Tsinghua Univ. (China)

INTERACTIVE PAPER SESSION: INDEXING AND RETRIEVAL

682224 A set-theoretic approach for compensated signature embedding using projections onto convex sets [6822-75]

S. Ababneh, R. Ansari, A. Khokhar, Univ. of Illinois at Chicago (USA)

682225 Semi-supervised dimensionality reduction for image retrieval [6822-76]

B. Zhang, IBM China Research Lab. (China); Y. Song, Tsinghua Univ. (China); W. Yin, M. Xie,

J. Dong, IBM China Research Lab. (China); C. Zhang, Tsinghua Univ. (China)

682226 Real-time image annotation by manifold-based biased Fisher discriminant analysis [6822-89]

R. Ji, H. Yao, J. Wang, X. Sun, X. Liu, Harbin Institute of Technology (China)

\section{INTERACTIVE PAPER SESSION: IMAGE/VIDEO CODING}

682227 Spectral coding of mesh geometry with a hierarchical set partitioning algorithm [6822-77]

U. Konur, Boğaziçi Univ. (Turkey); U. Bayazit, Istanbul Technical Univ. (Turkey);

H. F. Ateş, Işik Univ. (Turkey); F. S. Gürgen, Boğaziçi Univ. (Turkey) 
682228 Bitrate reduction techniques for stereoscopic digital cinema distribution [6822-78]

M. D. Smith, Consultant (USA); J. Villasenor, Univ. of California at Los Angeles (USA)

682229 Image modeling with parametric texture sources for design and analysis of image processing algorithms [6822-79]

C.-L. Chang, B. Girod, Stanford Univ. (USA)

6822 2A Unveiling relationships between regions of interest and image fidelity metrics [6822-80]

E. C. Larson, D. M. Chandler, Oklahoma State Univ. (USA)

6822 2B H.263 to VP6 video transcoder [6822-81]

C. Holder, H. Kalva, Florida Atlantic Univ. (USA)

INTERACTIVE PAPER SESSION: DISTRIBUTED SOURCE CODING

6822 2C Photoplus: auxiliary information for printed images based on distributed source coding [6822-82]

R. Samadani, D. Mukherjee, Hewlett-Packard Labs. (USA)

6822 2D Side information generation for distributed video coding based on optimal filtering [6822-83]

X. Zhang, Shanghai Conservatory of Music (China); J. Zhang, Univ. of Wisconsin, Milwaukee (USA)

6822 2E Enabling privacy for distributed video coding by transform domain scrambling [6822-84] M. Ouaret, F. Dufaux, T. Ebrahimi, Ecole Polytechnique Fédérale de Lausanne (Switzerland)

6822 2F Parameter selection for Wyner-Ziv coding of Laplacian sources [6822-85]

D. Mukherjee, Hewlett-Packard Labs. (USA)

Author Index 


\title{
Conference Committee
}

\author{
Symposium Chair
}

Nitin Sampat, Rochester Institute of Technology (USA)

Conference Chairs

William A. Pearlman, Rensselaer Polytechnic Institute (USA)

John W. Woods, Rensselaer Polytechnic Institute (USA)

Ligang Lu, IBM Thomas J. Watson Research Center (USA)

Program Committee

Tinku Acharya, Avisere, Inc. (USA)

Rashid Ansari, University of Illinois, Chicago (USA)

John G. Apostolopoulos, Hewlett-Packard Laboratories (USA)

Michel Barlaud, Université de Nice Sophia Antipolis (France)

Uluğ Bayazit, Işik University (Turkey)

Ali Bilgin, The University of Arizona (USA)

Mireille Boutin, Purdue University (USA)

Alan Conrad Bovik, The University of Texas, Austin (USA)

Maja Bystrom, Boston University (USA)

A. Enis Cetin, Bilkent University (Turkey)

Chang Wen Chen, Florida Institute of Technology (USA)

Qionghai Dai, Tsinghua University (China)

Gerard de Haan, Philips Research Laboratories (Netherlands)

Edward J. Delp, Purdue University (USA)

Eric Dubois, University of Ottawa (Canada)

Frederic Dufaux, École Polytechnique Fédérale de Lausanne (Switzerland)

Touradj Ebrahimi, École Polytechnique Fédérale de Lausanne (Switzerland)

Onur G. Guleryuz, DoCoMo Communications Laboratories USA, Inc. (USA)

Da-ke He, IBM Thomas J. Watson Research Center (USA)

Ashish Jagmohan, IBM Thomas J. Watson Research Center (USA)

Lina J. Karam, Arizona State University (USA)

Janusz Konrad, Boston University (USA)

C.-C. Jay Kuo, University of Southern California (USA)

Reginald L. Lagendijk, Technische Universiteit Delft (Netherlands)

Shipeng Li, Microsoft Research Asia (China)

Xin Li, West Virginia University (USA)

Jiebo Luo, Eastman Kodak Company (USA)

Enrico Magli, Politecnico di Torino (Italy) 
Michael W. Marcellin, The University of Arizona (USA)

Peyman Milanfar, University of California, Santa Cruz (USA)

Jens-Rainer Ohm, RWTH Aachen (Germany)

Thrasyvoulos N. Pappas, Northwestern University (USA)

Fernando Pereira, Instituto Superior Técnico (Portugal)

Beatrice Pesquet-Popescu, École Nationale Supérieure des

Télécommunications (France)

Fatih M. Porikli, Mitsubishi Electric Research Laboratories (USA)

Majid Rabbani, Eastman Kodak Company (USA)

Kenneth Rose, University of California, Santa Barbara (USA)

Amir Said, Hewlett-Packard Laboratories (USA)

Paul Salama, Indiana University-Purdue University at Indianapolis (USA)

Dan Schonfeld, University of Illinois at Chicago (USA)

Gaurav Sharma, University of Rochester (USA)

Eckehard G. Steinbach, Technische Universität München (Germany)

Robert L. Stevenson, University of Notre Dame (USA)

Thomas Stockhammer, Nomor Research (Germany)

Ming-Ting Sun, University of Washington (USA)

Andrew G. Tescher, AGT Associates (USA)

Bhaskaran Vasudev, Marvell Semiconductor, Inc. (USA)

Anthony Vetro, Mitsubishi Electric Research Laboratories (USA)

Zhou Wang, The University of Texas, Arlington (USA)

Xiaolin Wu, McMaster University (Canada)

Zixiang Xiong, Texas A\&M University (USA)

Yongyi Yang, Illinois Institute of Technology (USA)

Heather H. Yu, Panasonic Information and Networking Technologies Laboratory (USA)

Jun Zhang, University of Wisconsin, Milwaukee (USA)

Session Chairs

Media Over Networks

Bernd Girod, Stanford University (USA)

Indexing and Retrieval

Gaurav Sharma, University of Rochester (USA)

Scalable Video Coding

Onur G. Guleryuz, DoCoMo Communications Laboratories USA, Inc. (USA)

Video Coding

Amir Said, Hewlett-Packard Laboratories (USA)

Special Session: VLSI Architectures

Tinku Acharya, Avisere, Inc. (USA) 
Image Processing

Dan Schonfeld, University of Illinois at Chicago (USA)

Special Session: Color Demosaicking I

Xin Li, West Virginia University (USA)

Color Demosaicking II

Xin Li, West Virginia University (USA)

Special Session: Distributed Source Coding I

Ligang Lu, IBM Thomas J. Watson Research Center (USA)

Da-ke He, IBM Thomas J. Watson Research Center (USA)

Ashish Jagmohan, IBM Thomas J. Watson Research Center (USA)

Zixiang Xiong, Texas A\&M University (USA)

Special Session: Distributed Source Coding II

Ligang Lu, IBM Thomas J. Watson Research Center (USA)

Da-ke He, IBM Thomas J. Watson Research Center (USA)

Ashish Jagmohan, IBM Thomas J. Watson Research Center (USA)

Zixiang Xiong, Texas A\&M University (USA) 
Downloaded From: https://www.spiedigitallibrary.org/conference-proceedings-of-spie on 26 Apr 2023

Terms of Use: https://www.spiedigitallibrary.org/terms-of-use 